\title{
Parallel power paths and compactness of gear transmissions
}

\author{
K LAKSHMINARAYANA ${ }^{\dagger}$ \\ Mechanical Engineering Department, Indian Institute of Technology, Madras \\ 600036 , India
}

\begin{abstract}
Developments in mechanical power transmission have hinged around better materials and processes, new design ideas and effective new application of enduring scientific design principles and ideas. The current presentation aims at showing how deliberate measures to optimize load distribution have increased the compactness and capabilities of present day gear transmissions. Exploitation of parallel power paths, packing of gears in annular spaces and the effective use of epicyclic action by reducing the number of gear meshes and substituting rolling friction are explained.
\end{abstract}

Keywords. Mechanical power transmission; load distribution; parallel power paths; compact gear transmission.

\section{Introduction}

Transmissions serve primarily to convert and transmit motion and torque from a power source in a suitable manner for application. The source almost invariably provides uniform rotation. When it is converted into a non-uniform rotation, the device used is a nonuniform motion mechanism or a non-uniform transmission. Otherwise we have a uniform transmission or simply a transmission.

While there is a great variety of basically different transmission systems including hydrostatic transmissions and electric drives, we confine ourselves to purely mechanical systems here. In endeavouring to show how certain basic design principles play an important role in the modern development of compact transmissions, we confine ourselves mostly to gearing and indeed to only parallel-axis arrangements.

\section{Basic design objectives}

In transmitting power through a fairly high reduction, compactness is a basic requirement of steadily increasing importance. High efficiency then becomes essential even for small power units as the heat dissipation problem becomes more acute. 
There are many other objectives such as reliability, cost effectiveness, ease of maintenance, low noise levels, and ability to withstand critical environments. Keeping in view the basic aim of this presentation as stated in the introduction, we confine ourselves to the question of compactness and problems connected with the same.

\section{Load, stress and strength}

Out of this triplet of basic factors forming the foundation for compact designs, stress is decided mainly by form and size, while strength represents the role of material and treatments. Great care is taken to minimize the stress and maximize the strength. A very significant aspect of the load factor in realising the modern compact tranmissions is that of load distribution/equalization. This is however often lost sight of. In achieving a satisfactory level of load distribution, both a carefully developed application of design principles and the availability of sophisticated manufacturing facilities have played a pivotal role.

Design for effective load distribution thus becomes the main thrust of the current presentation. We limit ourselves to spur and helical gearing.

\section{Transverse distribution of load}

This refers to the distribution of tooth load between two or more pairs of gear teeth simultaneously in contact. Manufacturing accuracy (particularly in the form of low base pitch error) is naturally the first requirement for a good distribution of load. A high contact ratio (total contact ratio for helical gears) represents the theoretical occurrence of several simultaneous tooth-pair contacts. A large number of teeth, a large tooth height and a low pressure angle contribute to a high contact ratio. A large tooth height contributes to tip load leverage (for tooth root stress) but the tip load itself is reduced due to the high contact ratio. A more flexible tooth further helps in load distribution. A low pressure angle contributes to the basic all-load pitch-point contact stress but the pitch-point load itself may be reduced by the high contact ratio. The use of a large number of teeth on a given pitch cylinder is mainly limited by the bending strength since the tooth size is reduced. The tooth form however improves against the bending stress. Finally a large contact ratio generally reduces the noise level significantly.

Optimization of the number of teeth, pressure angle, tooth height and tooth profile modifications represents one way of improving the compactness of the basic spur gear pair. This qualitative discussion is to highlight the load distribution aspect.

\section{Longitudinal distribution of load}

This refers to the distribution of load along the length of the gear tooth. Manufacturing accuracy (particularly in the form of low tooth helix error) is naturally the first requirement for a good distribution of load.

In terms of practical requirements, compactness consists not only of low volume of space occupied but also involves achieving a low radial dimension, i.e. a low centre distance. This involves the permissibility of a relatively large face width. A larger face width however 


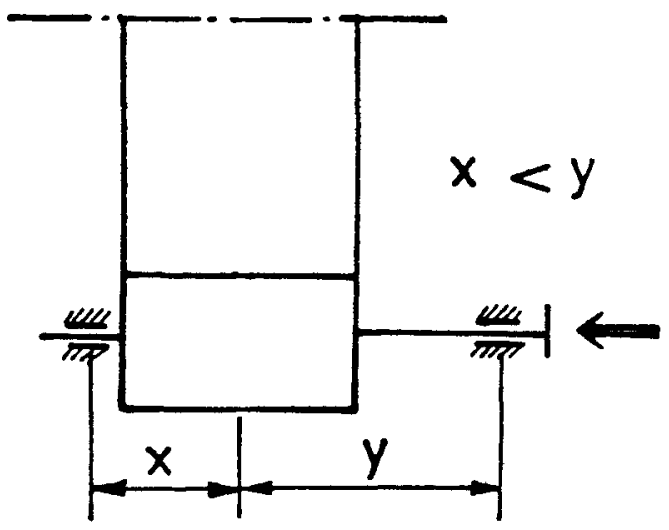

Figure 1. Straddle-mounted pinion situated near the bearing at the non-torque end of the shaft.

involves not only a possibly larger inaccuracy but also a larger bending and torsion of the pinion. Overhanging pinion is therefore avoided as far as possible and the pinion is straddle-mounted in bearings that are as close to the pinion as possible. The torsion effect on the pinion can be neutralised by the bending effect to some extent if the pinion is situated nearer the bearing at the non-torque end of the shaft. This is indicated in figure 1.

In the case of multistage gearing there is the possibility of a pinion on an intermediate shaft receiving power from both the ends. This is illustrated in figure 2. Maldistribution due to the torsion effect can be minimized in this fashion.

To improve the load distribution further, tooth helix modification has to be resorted to. This can be most advantageously used when the direction of major torque application is fixed and the magnitude of working load is fairly certain. The tooth helix correction may be made based on the most predominant load level or the average load. The correction for a symmetrically mounted pinion with one-sided torque application is illustrated in figure 3 . In practice, an approximation by a helix angle correction or a single-sided crowning will often suffice. When both faces of a tooth are being corrected, the middle one-third of the tooth length may be left uncorrected (i.e. straight for spur gears) or linearly corrected. This is to help tooth helix inspection, as shown in figure 4a. When only one side is corrected (e.g., lifting equipment), circular arc form crowning can be ground on that side as shown in figure $4 \mathrm{~b}$. The other side is left uncorrected for tooth helix inspection. The unsymmetrical

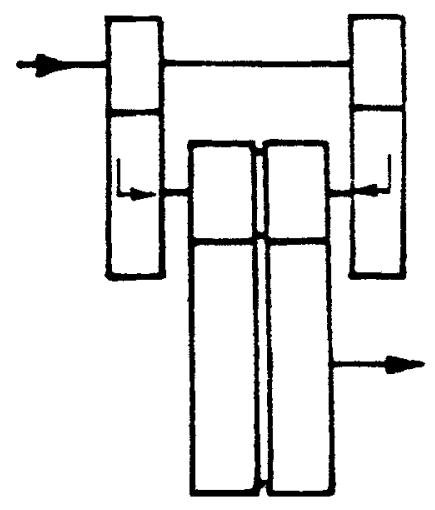

Figure 2. Pinion on an intermediate shaft receiving power from both the ends in multistage gearing. 


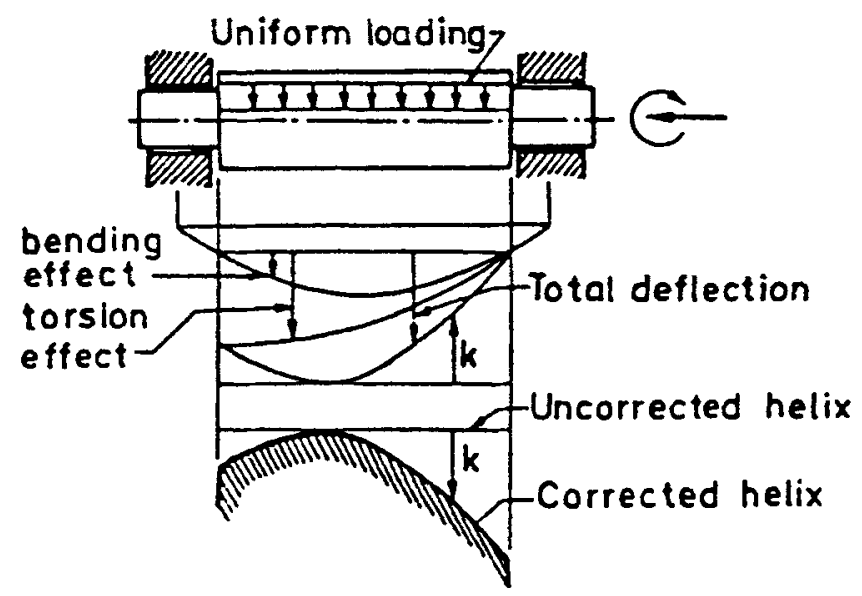

Figure 3. Illustration of correction for a symmetrically mounted pinion with one-sided torque application.

crowning in figure $4 \mathrm{~b}$ is the result of the approximate linear correction already referred to earlier and symmetrical crowning is introduced to counter manufacturing inaccuracy. In case of doubt it is better to go in for simple tooth-end relief since over-correction can prove to be detrimental.

\section{Load distribution between different gear pairs: Parallel power paths}

Figure 5 shows dual trandem gearing, also called the locked train. There are now two parallel power paths as shown in figure 6. Maldistribution of load between the two power paths can result from tooth errors (especially pitch errors), errors in the relative angular position of gears ( $3^{\prime}$ w.r.t. $2^{\prime}$ as against 3 w.r.t. 2 in figure 5), errors in the circumferential location of the intermediate gears as planets around the sun gears ( 1 and 4 in figure 5) or errors in $\delta_{1}$ and $\delta_{4}$ in figure 5 .

Figure 7 shows a simple planetary gear drive with three simple planets. The three parallel power paths are also shown in the same figure. Compactness is achieved by packing the annular space between the sun gear 1 and internal gear 3 with several planets. Up to 6 planets are used, obtainable speed ratio being reduced as the number of planets is increased. The

(a)

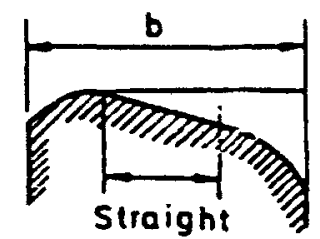

(b)

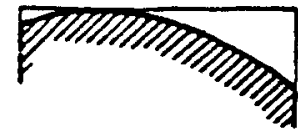

Figure 4. (a) Middle one-third of tooth length is left uncorrected for tooth helix inspection when both tooth faces are being corrected. (b) Only one side is corrected and circular arc form crowning is ground on that side. 


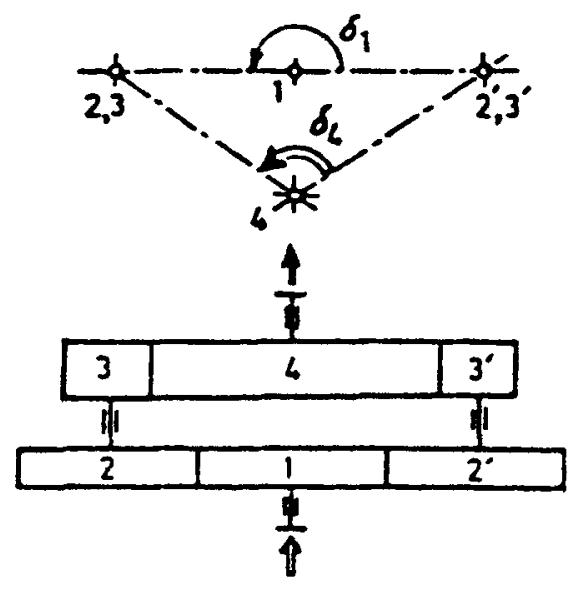

Figure 5. Dual tandem gearing, also called locked train.

compactness is however conditional on the achievement of an equitable distribution of load between the parallel power paths.

There are basically three different ways of proceeding towards load equalisation amongst parallel power paths:

(i) manufacturing accuracy,

(ii) flexibility, and

(iii) kinematically correct design (statical determinacy).

Some flexibility is always present, due to the elasticity of the machine elements. If there were no flexibility, only one power path would carry the entire load at any particular moment, depending on the relative magnitudes of the tooth spacing errors involved. Flexibility enables the gaps in the other power paths to close when the total load reaches a certain level. The additional load is carried equally by all the power paths.

Thus, with the same flexibility and ultimate load intensity, the lower the spacing errors, the more equitable is the distribution of load. Given the same spacing errors and the same ultimate load intensity, the higher the flexibility the more equitable is the load distribution amongst the parallel power paths. High flexibility, however, can cause vibration problems, beyond a point. It is therefore essential that a high degree of manufacturing accuracy be maintained, consistent with cost effectiveness. It may then be found, especially for small units, that the flexibility already present in the system is enough.

With a kinematically correct design, there is statical determinacy in the system. That is, the loads in the individual power paths are known (independent of the spacing errors which vary through a cycle and from unit to unit). The loads may not always be exactly equalized (this is further influenced by friction which is somewhat indeterminate).

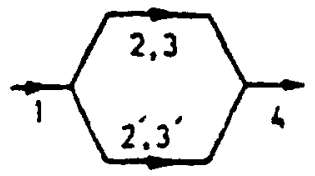

Figure 6. Illustration of two parallel power paths. 


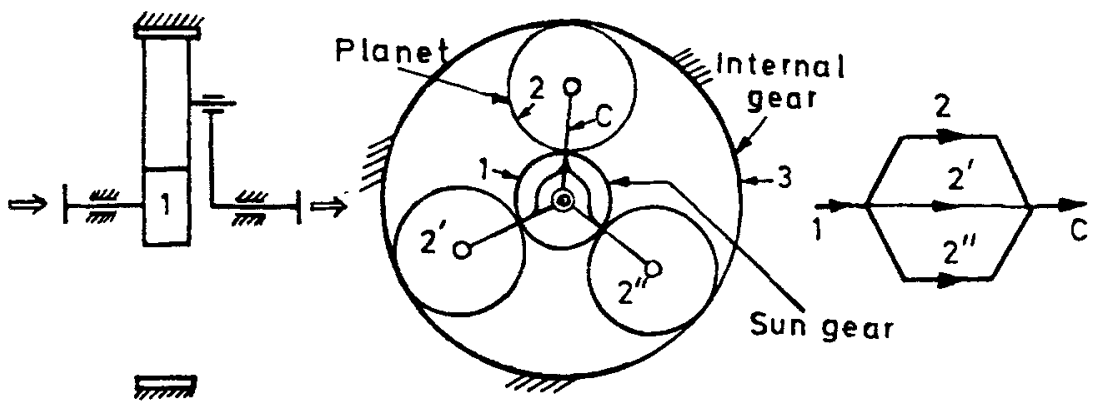

Figure 7. Schematic of a simple planetary gear drive with three planets. The three parallel power paths are also shown.

The standard kinematic solution to the load distribution problem for the three-planet drive of figure 7 is to 'float' the sun gear 1 . This is normally achieved by a gear coupling between the motor and the sun gear as shown symbolically in figure 8 . The coupling transmits a pure torque without imposing a lateral force on the sun gear 1. Ignoring friction, the three total tooth normal forces between the sun gear and the three planets are equalized when the planets are spaced at $120^{\circ}$ on the carrier. This load equalization is greatly dependent on effective lubrication of the gear coupling. This lubrication can easily fail since the internal movements in the coupling engendered by the varying tooth spacing errors in the gearing proper are rather small.

The sun gear centre moves in small loops, occasioned by the spacing errors. These movements can generate very significant inertia forces at high speeds with their dynamic effects on the meshes with the three planets. It is thus seen that a high degree of manufacturing accuracy is still essential.

Instead of the sun gear, the carrier may be 'floated' by providing a gear coupling at the output. The torque at the carrier being higher, the gear coupling will be larger.

Similarly, the internal gear may be floated. Coupling lubrication is then more involved since there is no rotation to help. Some proven high-power high-speed drives float both the sun and the internal gear. The inertia effects mentioned earlier are thus mitigated.

Kinematically correct designs for more than three planets have also been developed. Figure 9 shows a design for a 5-planet drive schematically. Here the carrier $\mathrm{C}$ is floated by means of a gear coupling at the output (coupling not shown). The sun gear is not floated.

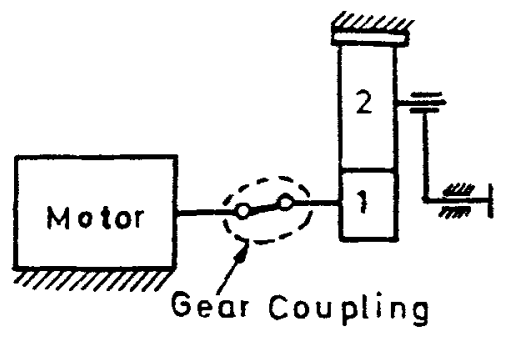

IPIP
Figure 8. Symbolic representation of gear coupling between the motor and the sun gear. 


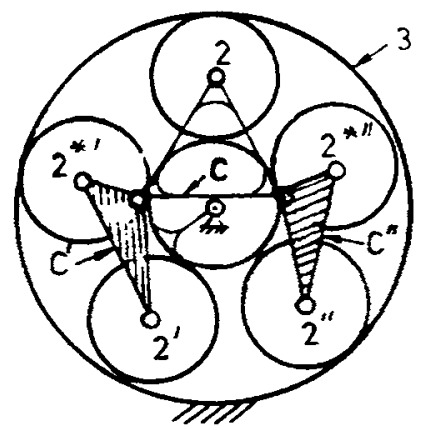

Figure 9. Schematic design for a 5-planet drive.

Auxiliary carriers $C^{\prime}$ and $C^{\prime \prime}$ carry two planets each. The dimensions of the carriers are adjusted to provide as equal a distribution of load as possible amongst the planets and ensure static balance. The planets are equally spaced.

The above and similar developments are based on the planar model. While theoretical studies have been made with the three-dimensional model, a practical approach to the third dimension is to use crowning on some of the gears.

Kinematically correct designs for the dual tandem gearing of figure 5 are now considered. Out of a large number of design possibilities, some are indicated below.

(i) Float the driving pinion (gear 1) by gear coupling.

(ii) Mount the driving pinion (gear 1) on a long lever.

(iii) Guide the bearing housing, in which the intermediate shafts are mounted linearly, perpendicular to centre distance.

(iv) Use helical teeth for gears 1,2, $2^{\prime}$. Make the two intermediate shafts $2-3$ and $2^{\prime}-3^{\prime}$ axially free. Balance the axial forces on these two shafts by means of springs or by means of the fluid connection as shown in figure 10.

(v) Introduce additional pinion $1^{\prime}$ with opposite helix (vis-a-vis 1 ) to mesh with $2^{\prime}$ and make the combined driving pinion $1-1^{\prime}$ axially free.

One of the guiding principles is to float only members with low inertia.

Some of the flexibility solutions to the load distribution problem are now indicated. In the dual tandem gearing of figure 5, torsionally flexible couplings may be interposed between gears 2 and 3 and between gears $2^{\prime}$ and $3^{\prime}$. Alternatively, the torsional flexibility

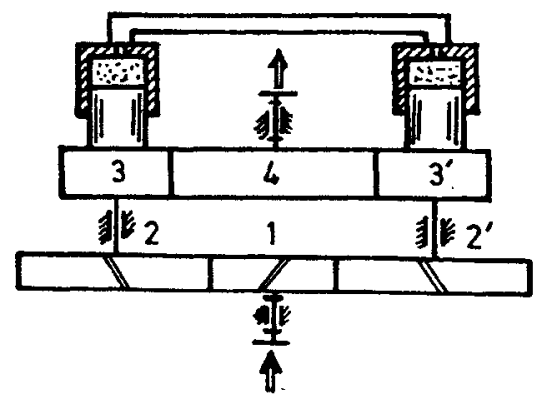

Figure 10. Balancing of axial forces on two axially free intermediate shafts by means of springs or a fluid connection. 


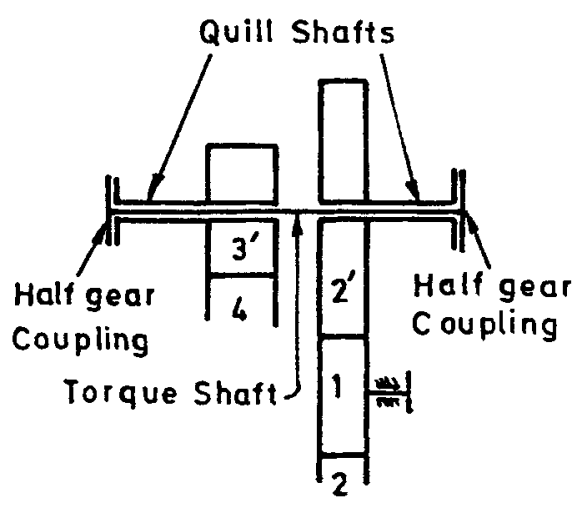

Figure 11. Increase of torsional flexibility of two intermediate shafts by means of the quill shaft design.

of the two intermediate shafts may be increased by means of the quill shaft design of figure 11. In the 3-planet drive of figure 7, the internal gear may be mounted on flexible material (or equivalent designs in metal).

\section{Assembly conditions for parallel power paths}

Closely linked with the statical indeterminancy of the parallel power path arrangements is the problem of assembly of gears of the additional power paths beyond the first. In figure 5, gear meshes 1-2, 3-4 and 1-2' can first be assembled without any problem. Gear $3^{\prime}$ can then be assembled with gear 4 only at particular angular positions of $3^{\prime}$ with respect to $2^{\prime}$. If one of these relative orientations is used, then the gears can be assembled. It is normally desired however to make units $2-3$ and $2^{\prime}-3^{\prime}$ of identical specifications. The angles $\delta_{1}$ and $\delta_{4}$ may then be suitably chosen or the number of teeth adjusted. In the planetary gearing of figure 7, the sum of the number of teeth on the sun gear and on the internal gear should be divisible by the number of planets, if the planets are to be equally spaced.

\section{Compactness through epicyclic action}

When a fairly high ratio of speed reduction is required, use of single-stage gearing will make the wheel very large. Two, three or more stages are thus introduced. The space requirement however can still be too large. Two or three simple planetary stages may be used and the coaxial input and output exploited. The compactness is otherwise due to the multiple planets in the individual stages.

In a simple epicyclic gear train consisting of two coaxial gear shafts $a, b$ and a carrier shaft $\mathrm{c}$, we can choose the tooth numbers such that the carrier-reference speed ratio $i_{\mathrm{ab}}$ is very near to $+1, b$ being fixed. We can then obtain an epicyclic speed reduction ratio of $I_{\mathrm{ca}}=1 /\left(1-i_{\mathrm{ab}}\right)$. If $i_{\mathrm{ab}}=1.02$, the speed reduction ratio from carrier $\mathrm{c}$ to co-axial gear a will be $=1 /(1-1.02)=-50$. Since the ratio $i_{\mathrm{ab}}$ is very nearly 1 , the tooth numbers are small and the drive is very compact for the large ratio demanded. The efficiency however goes down severely. With a tooth power loss factor of 0.005 , the efficiency will be only $66 \%$ and becomes just $50 \%$ if the tooth power loss factor is 0.01 . With a low efficiency, large 


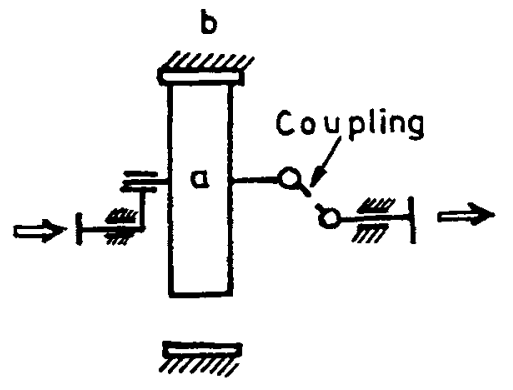

Figure 12. The 'open' train - reduction in the number of gear meshings from 2 to 1 in a power path.

heat is generated and the particularly compact unit is not equipped to dissipate the same. To improve the efficiency, one step is to reduce the number of gear meshes in a power path from 2 to 1 . The result is the 'open' train shown in figure 12. Here the absolute rotation of the planet is the output motion. This is 'brought down' to the axis of the co-axial unit by means of a constant velocity coupling using rollers. To obtain a large reduction, the number of teeth on the planet and internal gear differ by a small number, creating the problem of tip-to-tip interference. Special involute tooth proportions are needed. Using rollers in place of involute teeth on gear $b$ and a tooth difference of 1 , the tip-to-tip interference problem is circumvented by the rollers never leaving the mating planet. The demand for a low tooth power loss factor is simultaneously satisfied. (Due to rolling friction as well as low roller velocities; the latter are due to the internal-external mesh of almost +1 ratio.) This is the cyclo-drive system. Instead of the rollers being rotated about axes fixed on the ring gear (internal gear), they may be housed in a separate cage and interposed between concave pockets on the ring and the planet profile. This is the quadrant drive system. 